\title{
Electron Density Measurements of Argon Surface-Wave Discharges
}

\author{
M. Brake, ${ }^{1,2}$ J. Rogers, ${ }^{1,3}$ M. Peters, ${ }^{1}$ \\ J. Asmussen, ${ }^{1}$ and R. Kerber ${ }^{1}$
}

Received January 24, 1985; revised March 18, 1985

\begin{abstract}
The electron density of microwave-generated surface-wave discharges in argon have been measured using Stark broadening and calculated from the measured wavelengths of the standing surface wave. Results obtained from these two techniques compare well. The electron density varies from $10^{13}$ to $10^{14} / \mathrm{cm}^{3}$ for pressures ranging from 50 to 800 torr.
\end{abstract}

KEY WORDS: Microwave plasma; surface-wave discharge; Stark broadening; argon plasma.

\section{INTRODUCTION}

Experimental properties of microwave discharges produced by surfacewave launchers have been studied for several years. ${ }^{(1-3)}$ The electron density of low-pressure discharges ( $<10$ torr) has been determined by Langmuir probes, ${ }^{(3)} \mathrm{TM}_{010}$ microwave cavities, ${ }^{(1)}$ and by UHF interferograms of the surface wavelength. ${ }^{(1)}$ The electron density of an atmospheric-pressure discharge has been measured using Stark broadening. ${ }^{(2)}$ The objective of this paper is to summarize and compare measurements of spatially averaged electron densities $\left(N_{e}\right)$ made in argon, microwave-generated $(2.45 \mathrm{GHz})$ surface wave discharges. These new results span the intermediate pressure range of 50 to 800 torr, a pressure range not previously investigated, and compare two measurement methods: (1) electromagnetic measurements, similar to those traditionally used for low-pressure discharges and (2) Stark broadening, traditionally used for high-pressure discharges. The use of each

${ }^{1}$ College of Engineering, Michigan State University, East Lansing, Michigan 48824.

${ }^{2}$ Present Address: Department of Nuclear Engineering, University of Michigan, Ann Arbor, Michigan 48109.

${ }^{3}$ Present Address: MIT Lincoln Laboratory, Lexington, Massachusetts 02173. 
of these measurement techniques for the intermediate pressure regime will be discussed in the next section. The remainder of this introduction will give a qualitative characterization of the type of discharges investigated in this paper. Further descriptions can be found in Ref. 4.

The argon discharges can be described as weakly ionized, contracted, (i.e., separated from the tube wall by a nonionized gas layer), cylindrical plasma columns having diameters of $1-2 \mathrm{~mm}$ and lengths of $1-30 \mathrm{~cm}$ (depending on pressure and absorbed power). The discharges are visibly intense and have a clear and distinct boundary. Despite the short lengths, the discharges can be thought of as long plasma columns since the length-todiameter ratios are large. The discharges are maintained in steady state by an axially traveling surface wave, launched along the column by a microwave reentrant cavity. As the wave travels along the discharge column, the energy in the wave decays due to dissipation of microwave power in the discharge. Eventually a critical point is reached when the wave is cut off and the discharge physically ends. There are gradual axial variations in discharge diameter which can be directly related to the wave power at any axial point. ${ }^{(4)}$ In general, as microwave power is increased from 30 to $100 \mathrm{~W}$ at a constant pressure in a given discharge/tube configuration, plasma volume increases such that the spatially averaged density of absorbed power, $\langle P\rangle$, remains constant. ${ }^{(4)}$ In these experiments, the plasma volume increase is due to an increase in discharge length and a slight increase in discharge diameter.

\section{MEASUREMENT METHODS}

The experimental systems required to produce these argon surface-wave discharges are described in detail in Ref. 3. Figure 1 displays a typical arrangement with one surface-wave launcher and shows the orientation of the optical equipment, which was not used in the experiments of Ref. 3. Note that there is a gap between the center conductor and the flat cavity wall of the surface-wave launcher, allowing the coupling of microwave power to the discharges. Also, the plasma in Fig. 1 is shown filling the quartz tube radially. For the experiments of this paper, however, the plasma was always contracted to a smaller diameter $(1-2 \mathrm{~mm})$ than the quartz tube diameter.

In the first experiment, average electron densities were determined using electromagnetic analysis techniques to relate measurements to discharge properties. The standing surface wave configuration described in Ref. 3 was used with the discharges contained in a $4 \mathrm{~mm}$ i.d. quartz tube. In this experimental arrangement, two surface-wave launchers are used to sustain a single plasma column. The surface waves, which are traveling in 


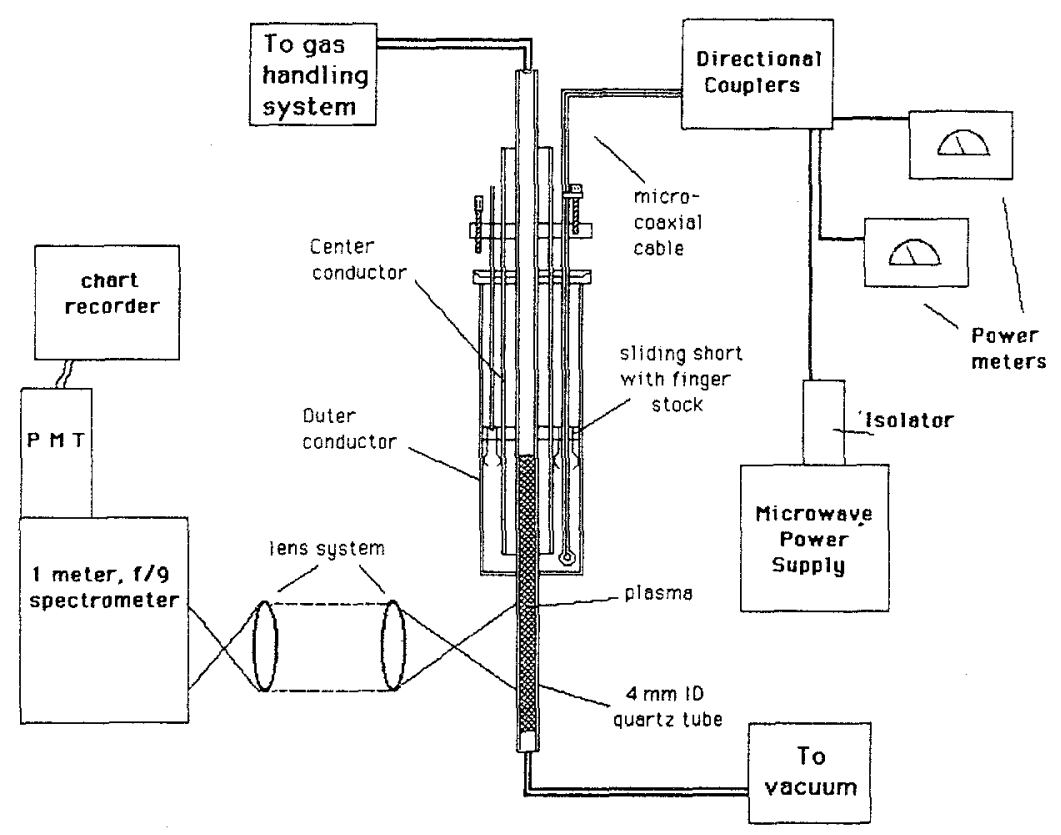

Fig. 1. Experimental schematic.

opposite directions, interact to form a standing wave in the region between the cavities. By using a movable E-field probe outside the discharge tube, ${ }^{3}$ the surface half wavelength can be measured. By modeling the discharge/quartz tube geometry as in Fig. 2, the measured half wavelength of the standing surface wave (typically $3.5-4.5 \mathrm{~cm}$ ) can be related to $N_{e}$ by a numerical solution to Maxwell's equations assuming: (1) cold plasma theory relates discharge complex conductivity to $N_{e}$ and effective collision frequency $\left(\nu_{e}\right)$, and (2) a reasonable value of $\nu_{e}$ is assumed. The procedure involved in the numerical solution is to find the complex roots of the transcendental equation resulting from application of boundary conditions at each interface (after writing Maxwell's equations in cylindrical coordinates in each region). ${ }^{(5)}$ The values of $\nu_{e}$ were obtained from a different experiment on similar argon discharges in which both $N_{e}$ and $\nu_{e}$ could be determined. The values assumed ranged from $1.0 \times 10^{10} \mathrm{sec}^{-1}$ to $3.0 \times$ $10^{10} \mathrm{sec}^{-1}$ over the pressure range of $50-800$ torr.

For the domain of these experiments, the calculated values of $N_{e}$ are not particularly sensitive to the assumed values of $\nu_{e}$; e.g., a change in $\nu_{e}$ by a factor of 10 might only change $N_{e}$ by a factor of 2 . Thus, the major uncertainty in these electromagnetic determinations of $N_{e}$ is how well the 


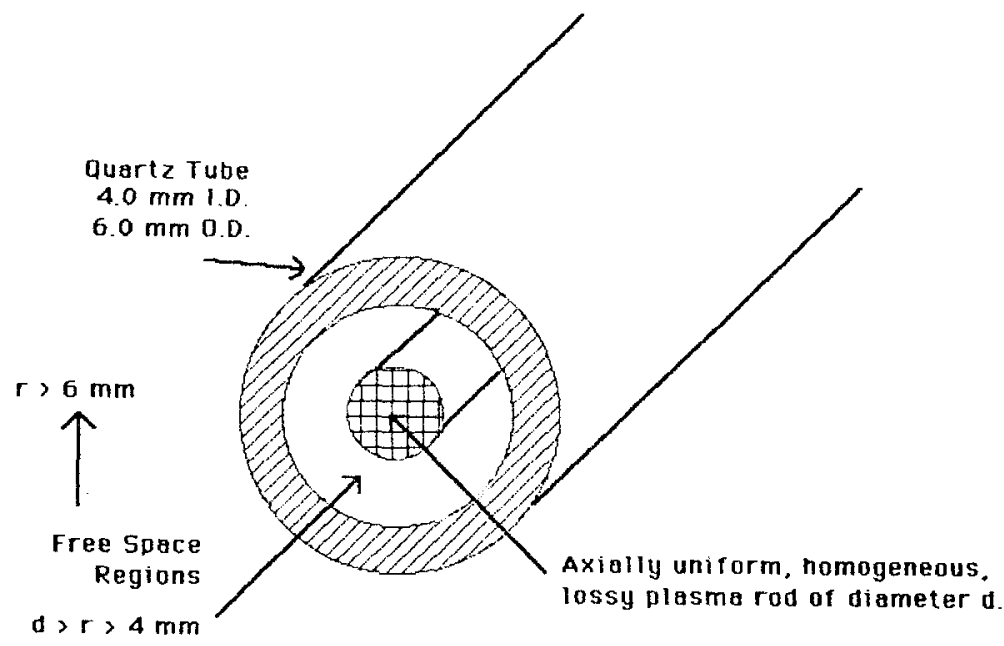

Fig. 2. Axially uniform, multilayered waveguide model for the discharge tube geometry. For calculations, $d$ was taken to be the measured average discharge diameter.

homogeneous discharge model averages out the inhomogeneities of the actual discharges. Analytically, it is difficult to assess how well the gradual axial variations in diameter are averaged by the measured average discharge diameter. However, the effect of radial electron density variations has been assessed analytically. Numerical comparisons between a radially inhomogeneous plasma column of the type considered here and a homogeneous one having $N_{e}$ equal to the average of the inhomogeneous case showed that the electric field distributions and dispersion relations between the two cases matched well. ${ }^{(4)}$ Thus, the dispersion characteristics of the surface wave are not sensitive to radial density profiles for the average densities and discharge diameters encountered in these experiments.

The second measurement of $N_{e}$ involved emission spectroscopy. Ideally, this technique could have been used simultaneously with the first method, but logistics prevented this. Thus, for the second set of experiments, the argon surface-wave discharges were recreated as best as possible at the same experimental conditions of gas pressure and absorbed power density using one surface-wave launcher (as in Fig. 1). The emitted light from the plasma (approximately a few centimeters in length and a few millimeters in width) was imaged onto the entrance slit of a $1-\mathrm{m}, f / 9$ grating spectrometer. A high-quantum-efficiency photomultiplier tube connected to a chart recorder was used to detect the light signal. The hydrogen $\mathrm{H} \beta(4861 \AA)$ line was observed due to trace amounts of water vapor in the gas-handling system. If additional amounts of $\mathrm{H}_{2}$ were added to the plasma in an attempt 
to improve the signal-to-noise ratio, the plasma properties were observed to change. The length of the plasma was shortened and it required considerably more power to sustain the plasma. The full width at half height (fwhh) of $\mathrm{H} \beta$ was measured for several pressures at zero flowrate and at a flowrate of $5 \mathrm{ml} / \mathrm{sec}$.

The following method was used to determine the electron density from the amount of Stark broadening observed in $\mathrm{H} \beta$. A gas temperature of $1000 \mathrm{~K}$ (based upon computer modeling of the chemical kinetics) ${ }^{(6)}$ was used to determine the Doppler contribution to the $H \beta$ fwhh. The Doppler contribution was small, $0.07 \AA$. The argon $4300 \AA$ line, which has a negligible amount of Stark broadening, was used to determine the instrument broadening. The fwhh of the argon $4200 \AA$ line was $0.22 \AA$ which was found to be the overall resolution of the optical system using a low-pressure discharge lamp under similar optical conditions. Using a computer fit, the argon $4200 \AA$ line was found to be slightly better described by a Gaussian shape than a Lorentzian shape. For the purpose of finding the fwh of the Stark-broadened portion of $H \beta$, the Stark line shape was assumed to be approximated by a Lorentzian. ${ }^{(7,8)}$ Tables of Voight profiles ${ }^{(9)}$ were used to determine the Gaussian $\left(\sqrt{\text { Doppler }^{2}+\text { Instrument }^{2}}\right)$ and the Lorentzian fractions of the fwhh of $\mathrm{H} \beta$. The $N_{e}$ was calculated using Griem's formula, ${ }^{(7)}$ assuming an electron temperature of $10,000 \mathrm{~K}$ (based on atomic boltzmann plots) ${ }^{(6)}$ and an electron density of $10^{14} \mathrm{~cm}^{-3}$.

The method of finding electron densities from Stark-broadening measurements found only spatially average values $(1-3 \mathrm{~cm}$ discharge length, see Fig. 1) as did the previous method, and this method was only useful for pressures greater than 30 torr. Lower pressures had negligible amounts of Stark broadening compared to instrument broadening. Even though the electron temperature may change from 5000 to $15,000 \mathrm{~K}$ as the experimental pressure is varied, the resulting error in the electron density is at most a few percent. The error resulting from using one estimated electron temperature and density in Griem's formula, as well as the error in assuming that the Stark profile was Lorentzian, is much smaller than the error resulting from the estimated line shape of the instrument function. If a Lorentzian line shape is assumed for the instrument function, the average electron densities are reduced by about $50 \%$. This uncertainty is reflected in the representative error bars of Figs. 3 and 4.

\section{RESULTS}

Figure 3 compares the two methods of measuring the electron density by displaying $N_{e}$ vs. pressure. The two methods agree reasonably well with the spectroscopic method giving results about a factor of 2 higher than the 


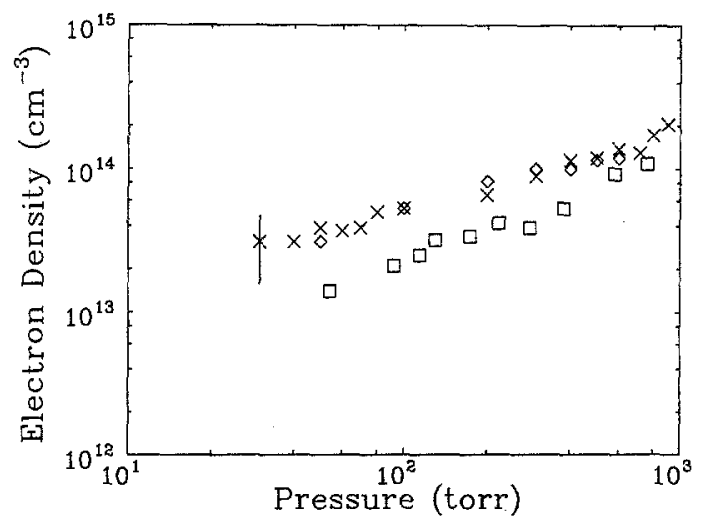

Fig. 3. The electron density as a function of pressure as determined by Stark broadening at flowrates of $5 \mathrm{ml} / \mathrm{sec}(x)$ and $0 \mathrm{ml} / \mathrm{sec}(\diamond)$ and by the EM method for a flowrate of $0 \mathrm{ml} / \mathrm{sec}(\square)$.

EM method. Figure 4 displays $N_{e}$ vs. absorbed power and shows that both measurement methods indicate that $N_{e}$ is constant with absorbed power at a constant pressure. This behavior of $N_{e}$ with absorbed power is similar to that of $\langle P\rangle$ described in the Introduction. Thus, the ratio of $\langle P\rangle$ to $N_{e}$ for these discharges may be a constant at a constant pressure, similar to low-pressure discharges.

The results shown in Figure 3 support the use of these two methods for measuring $N_{e}$ in microwave discharges for the pressure regime of

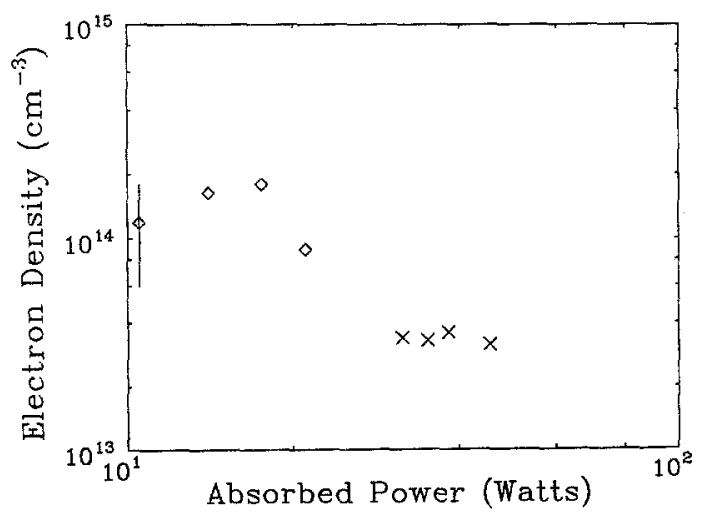

Fig. 4. The $N_{e}$ as a function of absorbed power as determined by Stark broadening at a pressure of 300 torr $(\diamond)$ and the EM method at a pressure of 174 torr $(\times)$. 
50-800 torr. However, the potential sources of error and underlying assumptions in each method must be analyzed further.

\section{ACKNOWLEDGMENT}

This work was supported by NASA Grant No. 3299.

\section{REFERENCES}

1. M. Moison, C. Beaudry, and P. Leprince, "A New HF Device for the Production of Long Plasma Columns at a High Electron Density," Phys. Lett. A 50, 125-126 (1974).

2. J. Hubert, M. Moison, and A. Ricard, "A New Microwave Plasma at Atmospheric Pressure," Spectrochim. Acta B 34, 1-10 (1979).

3. J. Rogers and J. Asmussen, "Standing Waves along a Microwave-Generated Surface Wave Plasma," IEEE Trans. Plasma Sci. 10, 11-16 (1982).

4. J. Rogers, "Properties of Steady-State, High-Pressure, Argon Microwave Discharges," Ph.D. Thesis, Michigan State University (1982).

5. R. F. Harrington, Time Harmonic Electromagnetic Fields, McGraw-Hill, New York (1961), Chapter 5.

6. M. Brake, "A Theoretical and Experimental Investigation of the Chemical Kinetics of an Oxygen Microwave Discharge," Ph.D. Thesis, Michigan State University (1983), Appendix D.

7. H. Griem, Plasma Spectroscopy, McGraw-Hill, New York (1964), p. 538.

8. W. Weise, "Line Broadening," in Plasma Diagnostic Techniques, R. Huddlestone and S. Leonard, eds., Academic Press, New York (1965).

9. J. Davies and J. Vaughan, "A New Tabulation of the Voight Profile," Astrophys. J. 137, 1302-1305 (1963). 